\title{
AVALIAÇÃO DA TRAJETÓRIA MÉDIA DE CRESCIMENTO DE CAPRINOS EM MODELOS DE REGRESSÃO ALEATÓRIA*
}

\author{
EVALUATION OF AVERAGE GROWTH CURVE OF GOATS USING \\ RANDOM REGRESSION MODEL
}

\author{
Sousa, J.E.R. ${ }^{1 A}$, Silva, M.A. ${ }^{2}$, Sarmento, J.L.R. ${ }^{1}$, Sousa, W.H. ${ }^{3}$ e Souza, M.S.M. ${ }^{1}$
}

${ }^{1}$ CPCE/UFPI. Av. Adelmar Moreno Benvindo. Caixa postal, 72. CEP 64900-000 Bom Jesus. PI. Brasil. Aernandes@ufpi.br

${ }^{2}$ Escola de Veterinária. DZ. UFMG. Brasil.

${ }^{3}$ EMEPA-PB. Brasil.

\section{PalaVRas chaVe ADICIONAIS}

Anglo-Nubiana. Curva de crescimento. Função polinomial. Regressão fixa.

\section{RESUMO}

Avaliou-se a utilização de funções polinomiais com ordem de ajuste variando de dois a sete na descrição da curva média de crescimento (Regressão Fixa) e sua influência sobre os parâmetros genéticos de 946 caprinos da raça Anglo-Nubiana, criados entre 1980 e 2005. Para modelar a regressão fixa, os modelos polinomiais da idade foram comparados quanto à adequação do ajuste da curva média de crescimento empregando-se os seguintes critérios: quadrado médio do resíduo, desvio médio absoluto, percentagem quadrada de viés e coeficiente de determinação. Os critérios de informação de Akaike, Bayesiano de Schwarz e logaritmo da função de máxima verossimilhança foram utilizados para verificar a influência das diferentes ordens de ajustes da regressão fixa sobre a estimativa dos parâmetros genéticos. A melhoria mais expressiva no ajuste ocorreu até a ordem quatro, sugerindo que esta foi suficiente para modelar a curva média de crescimento da população em estudo. Pouca influência sobre a estimativa dos parâmetros genéticos foi observada com adoção de funções superiores à cúbica. A herdabilidade direta apresentou comportamento crescente até 84 dias de idade, enquanto que a materna manteve-se praticamente constante durante todo período analisado. Com base nos resul-

* Parte da tese de Doutorado do primeiro autor em Ciência Animal. Escola de Veterinária/UFMG.

\section{AdDitional KeYWORDS}

Anglo-Nubian. Growth curve. Polynomial function. Fixed regression.

tados do conjunto de critérios utilizados, os polinômios ortogonais da idade mostraram-se bastante eficientes e polinômios com pelo menos ordem quatro devem ser empregados para representar a curva média de crescimento em modelos de regressão aleatória.

\section{SUMMARY}

Polynomial functions for modeling growth curves of 946 Anglo-Nubiano goats, born from 1980 to 2005 , and their effect on genetic parameter estimates were evaluated. Growth trajectories were fitted by fixed regression of weights on orthogonal polynomial of age, considering polynomials from second to seventh orders. For fixed regression, polynomial models were compared using the following criteria: mean square errors, absolute average residual error, square percent bias and goodness of fit. Akaike's information criterion, Bayesian information criterion and likelihood ratio test criteria were used to evaluate the effect of different polynomial order of fixed regression on genetic parameters estimates. An expressive improvement was found using higher order polynomials and those of fourth order were sufficient for modeling the average growth curve of goats. No influences on genetic parameter estimates were observed adopting higher than cubic order polynomial functions. The direct heritability showed an increasing pattern until $84^{\text {th }}$ 
day of age, while the maternal one had a constant pattern for the whole period. Polynomial functions are efficient tools for modeling growth curve and polynomials of at least fourth order should be used for modeling the average growth curve of goat in random regression models.

\section{INTRODUÇÃO}

Os modelos de regressão aleatória, utilizados nas avaliações genéticas de animais, empregam funções lineares contínuas para descreverem tanto os efeitos fixos como os efeitos aleatórios, sendo os valores genéticos preditos por funções contínuas dos desvios de cada animal, tomados como aleatórios, em relação a uma curva média, considerada como efeito fixo. Nos estudos de crescimento, a função contínua da parte fixa dos modelos de regressão aleatória é conhecida como trajetória média de crescimento, por representar a tendência de crescimento do peso corporal médio da população em relação às diferentes idades. (Kirkpatrick et al., 1990). Apesar de sua relevante importância, muito pouco tem sido feito em relação a parte fixa da regressão, sendo que, em alguns estudos, a parte fixa têm sido modelada com base em informações da literatura ou de acordo com a modelagem da parte aleatória, o que pode não ser a melhor estratégia de análise.

Em bovinos de corte, alguns autores investigaram a ordem de ajuste de funções polinomiais para representar a curva média de crescimento em modelos de regressão aleatória. Meyer (1999) estudou o crescimento pós-desmame de fêmeas de corte de duas raças bovinas na Austrália por meio de polinômios ortogonais da idade de várias ordens, empregando o método de quadrados mínimos ordinários, ignorando o efeito de animal. A autora comparou funções com ordens variando de dois até oito e concluiu que o crescimento dos animais foi melhor descrito por uma regressão cúbica. No Brasil, Sakaguti et al. (2002) averiguaram a ordem mais apropriada de funções polinomiais da idade para descrever a curva média de crescimento de bovinos Tabapuã. Estes autores também compararam diferentes ordens em análises de quadrados mínimos ordinários e concluíram que a curva média de crescimento deve ser representada por polinômios de, pelo menos, quarta ordem. Arango et al. (2004) empregaram o procedimento de modelos mistos para encontrar a melhor ordem de ajuste da regressão fixa sobre a idade para modelar a trajetória média da população. Segundo estes autores a regressão quadrática da idade foi a ordem polinomial mais alta com efeito significativo, o que foi determinante para os autores assumirem como a ordem de melhor ajuste.

Sarmento et al. (2006), ao estudarem a curva de crescimento de ovinos Santa Inês, utilizaram polinômios ortogonais de Legendre de ordens três e quatro no ajuste do modelo como um todo, ou seja, da trajetória média de crescimento e das regressões aleatórias; os autores concluíram que o modelo empregando uma função contínua cúbica proporcionou melhor ajuste. Entretanto, modelar a curva fixa da mesma forma que a parte aleatória pode não ser a melhor estratégia de análise.

Em caprinos, ainda são poucos os trabalhos que empregam regressão aleatória para avaliação genética. Destacam-se os trabalhos de Assis et al. (2006) e Breda et al. (2006) em que avaliaram a curva média de lactação em cabras leiteiras da raça Parda Alpina. No que se refere as características de crescimento a literatura é ainda mais limitada, sendo o trabalho de Sousa (2007), sobre crescimento de caprinos da raça Anglo-Nubiana um dos poucos encontrados.

Este trabalho avaliou a utilização de funções polinomiais na descrição da curva média de crescimento de caprinos da raça Anglo-Nubiana para estabelecer o melhor ajuste dos efeitos fixos em avaliações genéticas utilizando modelos de regressão aleatória, bem como verificar a influência de diferentes ordens de ajuste da parte fixa dos modelos de regressão aleatória nas estimativas de parâmetros genéticos. 


\section{AVALIAÇÃO DA TRAJETÓRIA MÉDIA DE CRESCIMENTO DE CAPRINOS}

\section{MATERIALEMÉTODOS}

\section{DESCRIÇÃODOSDADOS}

Foram utilizados registros de produção de caprinos Anglo-Nubiano no período de 1980 a 2005, provenientes da Fazenda Experimental Pendência, pertencente à Empresa Estadual de Pesquisa Agropecuária da Paraíba (EMEPA - PB). A Fazenda situa-se no município de Soledade, na região dos Cariris Velhos Paraibanos, correspondendo a uma área de 739 ha à $546 \mathrm{~m}$ de altitude, distante $210 \mathrm{~km}$ de João Pessoa. A média de temperatura máxima anual é $35^{\circ} \mathrm{C}$ e mínima $22^{\circ} \mathrm{C}$, com pequenas variações. A umidade relativa do ar média é de $50 \%$ com pluviosidade média de $390 \mathrm{~mm} / \mathrm{ano}$, que varia de 105 a $705 \mathrm{~mm} / \mathrm{ano}$.

Após edição dos dados, havia 4313 observações de peso, do nascimento aos 196 dias, sendo que as pesagens eram intercaladas por períodos médios de 28 dias. Os pesos corporais foram distribuídos em seis classes de idade, sendo a primeira classe constituída pela pesagem ao nascimento, a segunda pelos pesos aos 28 e 56 dias, terceira por pesos aos 84 e 112 dias, quarta pelos pesos aos 140 dias, quinta pelos pesos aos 168 dias, e a sexta pelos pesos aos 196 dias de idade; em dois sexos: machos e fêmeas; em três tipos de nascimento: simples, duplos e triplos e em duas estações de pesagens: chuvosa (de março a julho) e seca (de agosto a fevereiro). Foram ainda mantidos no arquivo grupos de contemporâneos (ano e estação de pesagem e classe de idade) com no mínimo três observações. As classes de idade, foram incluídas nos grupos de contemporâneos, para diminuir a amplitude de idades dos animais comparados diretamente dentro de cada grupo.

\section{Práticas de MANEJo}

Os animais foram criados em sistema semi-intensivo alimentados em piquetes de pastagem nativa e nativa melhorada. Receberam suplementação alimentar à base de silagem de milho (Zea mays L.) e sorgo
(Sorghum bicolor (L.) Moench), feno de maniçoba (Manihot glaziowii Mull.), palma forrageira (Opuntia ficus sp.), e ração concentrada com 14 - 16\% de proteína, quando necessário. A suplementação mineral foi prática constante durante todo o ano. $\mathrm{O}$ plano sanitário consistia de vacinações, controle sistemático de endo e ectoparasitas e tratamento clínico da linfadenite caseosa.

O rebanho era submetido a dois períodos de monta controlada, com duração de 40 a 60 dias, que, normalmente, tinham início nos meses de março e novembro, fazendo com que as parições se concentrassem nos períodos de fevereiro a abril e de agosto a setembro. Durante as estações de monta, as cabras recebiam suplementação alimentar em razão da escassez de forragem na região. As cabras em estro eram identificadas por meio de machos vasectomizados e a monta era controlada a intervalos de, aproximadamente, 12 horas, até a não aceitação do reprodutor pela cabra. Os reprodutores eram utilizados por período máximo de três anos consecutivos, sendo então removidos do rebanho. As cabras eram utilizadas por, no máximo, sete anos, podendo ser descartadas, também, por insucesso na fertilidade ao parto, por pouca habilidade materna e por enfermidades.

Os animais eram separados da mãe ao nascimento, recebiam colostro três vezes ao dia, a partir do 10 dia de vida recebiam dieta sólida e eram desaleitados aos 70 dias de vida. Esse manejo era adotado principalmente como forma preventiva da ArtriteEncefalite Caprina (CAE).

\section{ANÁlisesdos dAdos}

Análises preliminares foram realizadas para ajustar a trajetória média da população por meio de uma regressão fixa utilizando polinômios ortogonais da idade, com ordem de ajuste que variaram de dois a sete, ou seja, $(\mathrm{k}=2, \ldots, \mathrm{k}=7)$. As análises foram executadas por meio do método dos quadrados mínimos ordinários, utilizando o procedimento GLM do SAS Institute (1999), 
ignorando o efeito de animal. Os efeitos fixos de grupo contemporâneo, sexo e tipo de nascimento da cria e o efeito linear da covariável idade da cabra ao parto foram considerados no modelo. Análises indicaram que o efeito quadrático da covariável idade da cabra ao parto não foi significativo, desta forma, este efeito não foi incluído no modelo.

Os diferentes modelos polinomiais foram comparados quanto à adequação de ajuste da curva média de crescimento empregando os seguintes critérios: quadrado médio do resíduo (QMR), obtido por:

$$
Q M R=\frac{1}{n} \sum_{i=1}^{n}\left(y_{i}-\hat{y}_{i}\right)^{2},
$$

em que:

n é o número de observações,

$y_{i}$ e $\hat{y}_{i}$ são os valores observados e estimados, respectivamente;

desvio médio absoluto (DMA), que é dado por:

$$
D M A=\frac{\sum_{i=1}^{n}\left|y_{i}-\hat{y}_{i}\right|}{n},
$$

utilizado por Sarmento et al. (2006), obtido como o somatório dos desvios médios em valores absolutos; percentagem quadrada de viés (PQV), proposto por Ali e Schaeffer (1987), determinado por:

$$
P Q V=\frac{\sum_{i=1}^{n}\left(y_{i}-\hat{y}_{i}\right)^{2}}{\sum_{i=1}^{n}\left(y_{i}\right)^{2}} x 100,
$$

e o coeficiente de determinação $\left(\mathrm{R}^{2}\right)$, obtido por meio do quadrado da correlação entre os pesos observados e estimados por uma função, que equivale à:

$$
1-\left(\frac{S Q R}{S Q T_{c}}\right),
$$

em que:

SQR é a soma de quadrados do resíduo e,
SQTC a soma de quadrados total corrigida pela média.

Em um segundo momento, visando avaliar a influência da ordem de ajuste empregada para representar a trajetória média da população sobre os componentes de variância e parâmetros genéticos, ajustaram-se diferentes modelos de regressão aleatória, variando-se a ordem de ajuste da regressão fixa de dois a sete e mantendose constante a parte aleatória com ajuste de ordem três. Tanto as regressões fixas como aleatórias foram representadas por funções contínuas, cujas idades foram descritas em termos de polinômios ortogonais de Legendre.

O modelo estatístico geral pode ser representado da seguinte forma:

$$
\begin{aligned}
& y_{i j}=F_{i j}+\sum_{m=0}^{k \beta-1} \beta_{m} \phi_{m}+\sum_{m=0}^{k a-1} \alpha_{i m} \phi_{m}+ \\
& \sum_{m=0}^{k m-1} \gamma_{i m} \phi_{m}+\sum_{m=0}^{k c-1} \delta_{i m} \phi_{m}+\xi_{i j}
\end{aligned}
$$

em que:

$y_{i j}$ é o peso j da cria i; $\phi_{m}$ é a função polinomial de Legendre $m$ da idade padronizada $(-1 a+1) ; F_{i j}$ é um conjunto de efeitos fixos incluídos no modelo (grupo de contemporâneos, sexo e tipo de nascimento da cria e pela covariável linear da idade da cabra ao parto ; $\beta_{\mathrm{m}}$ são os coeficientes de regressão fixos do peso sobre o polinômio de Legendre, com $\mathrm{k}_{\beta}$ variando de dois a sete $\left(\mathrm{k}_{\beta}=2, \ldots, \mathrm{k}_{\beta}=7\right)$ para modelar a trajetória média da população; $\alpha_{i m}, \gamma_{i m}$ e $\delta_{\text {im }}$ são os coeficientes de regressão aleatória genético aditivo direto, genético aditivo materno e de ambiente permanente de animal, $\mathrm{k}_{\mathrm{a}}=3, \mathrm{k}_{\mathrm{m}}=$ 3 e $k_{c}=3$ são as ordens dos polinômios a serem ajustados; $\varepsilon_{\mathrm{ij}}$ é o efeito aleatório residual.

Em notação matricial, o modelo acima e suas respectivas pressuposições podem ser descritos como:

$$
\mathrm{Y}=\mathrm{X} \beta+\mathrm{Z}_{1} \alpha+\mathrm{Z}_{2} \gamma+\mathrm{W} \delta+\varepsilon, \mathrm{e}
$$




\section{AVALIAÇÃO DA TRAJETÓRIA MÉDIA DE CRESCIMENTO DE CAPRINOS}

$$
E\left(\begin{array}{l}
y \\
\alpha \\
\gamma \\
\delta \\
\varepsilon
\end{array}\right)=\left(\begin{array}{c}
X \beta \\
0 \\
0 \\
0 \\
0
\end{array}\right) e\left\{\begin{array}{l}
\operatorname{Var}(\alpha)=\mathrm{K}_{\mathrm{a}} \otimes \mathrm{A}, \\
\operatorname{Var}(\gamma)=\mathrm{K}_{\mathrm{m}} \otimes \mathrm{A}, \\
\operatorname{Var}(\delta)=\mathrm{K}_{\mathrm{c}} \otimes \mathrm{I}, \\
\operatorname{Var}(\varepsilon)=\mathrm{R} ;
\end{array}\right.
$$

em que:

y é o vetor de observações, $\beta$ o vetor de efeitos fixos (incluindo $F_{i j}$ e $\beta_{m}$ ), $\alpha$ o vetor aleatório dos coeficientes genéticos aditivos diretos, $\gamma o$ vetor aleatório dos coeficientes genéticos aditivos maternos, $\delta$ o vetor de coeficientes de ambiente permanente de animal, $X, Z_{1}, Z_{2}, W$, são as matrizes de incidência correspondentes e $\varepsilon$ o vetor de resíduos. $\mathrm{K}_{\mathrm{a}}, \mathrm{K}_{\mathrm{m}}$ e $\mathrm{K}_{\mathrm{c}}$ são as matrizes de variâncias e covariâncias entre os coeficientes de regressão aleatórios para os efeitos genéticos aditivos direto e materno e de ambiente permanente de animal, respectivamente, Ré a matriz de variâncias residuais. A é a matriz do numerador do coeficiente de parentesco aditivo, I é uma matriz identidade $\mathrm{e} \otimes$ o produto direto.

A variância residual foi assumida como homogênea ao longo de todas as idades e a covariância entre os efeitos genéticos direto e materno foi considerada igual a zero. Os componentes de variâncias e covariâncias entre os coeficientes de regressão aleatórios foram estimados pelo método da máxima verossimilhança restrita (REML), utilizando-se a opção DXMRR do pacote estatístico DFREML (Meyer, 1998) e empregando o algoritmo livre de derivadas para maximizar o log da função de verossimilhança.

Modelos de regressão aleatória com diferentes ordens de ajustes para a regressão fixa foram comparados pelo logaritmo da função de máxima verossimilhança $(\log L)$, que permite comparações apenas entre modelos aninhados e tende a favorecer modelos com maior número de parâmetros. Assim foram usados também os critérios de informação de Akaike (AIC) e Bayesiano de Schwarz (BIC), que permitem comparações entre modelos não-aninhados e impõem penalidades de acordo com o número de parâmetros a serem estimados. Para AIC e
BIC, o valor para comparação é obtido conforme a seguir:

$$
\begin{aligned}
& A I C=-2 \ln L+2 p e \\
& B I C=-2 \ln L+p \ln (N-r(X))
\end{aligned}
$$

em que:

p é o número de parâmetro estimado; $\mathrm{N}$ o número total de observações; e r (X), o posto da matriz de incidência dos efeitos fixos no modelo.

\section{RESULTADOSEDISCUSSÃO}

Análises preliminares, obtidas por meio dos quadrados mínimos ordinários ignorando-se os efeitos aleatórios para diferentes ordens de ajustes da trajetória média da população, estão apresentadas na tabela $\mathbf{I}$.

Observa-se (tabela I) que houve melhoria de ajuste na comparação do modelo um com o dois, e do dois com o três, conforme os valores de QMR, DMA, PQV e R ${ }^{2}$. Comparando os polinômios da quinta a sétima ordem, observou-se que praticamente não houve mudança ou em escalas muito pequenas, refletindo em pouca melhoria com a adoção de ordens superiores a quatro, o que sugere uma função cúbica, como a que apresenta melhor ajuste para a curva média de crescimento. Resultados semelhantes

Tabela I. Quadrado médio do residuo (QMR), desvio médio absoluto (DMA), percentagem quadrada de viés (PQV) e coeficiente de determinação $\left(R^{2}\right)$ para as diferentes ordens de ajustes ( $k$ ) da trajetória populacional de crescimento. (Mean square errors (QMR), absolute average residual error (DMA), square percent bias (PQV) and goodness of fit $\left(R^{2}\right)$ for different order of adjust of Growth trajectorie).

\begin{tabular}{lcrrr}
\hline Modelo & QMR & DMA & PQV (\%) & $\mathrm{R}^{2}$ \\
\hline 1- k=2 & 9,4565 & 2,2784 & 5,9631 & 0,7592 \\
2- k=3 & 8,8002 & 2,1086 & 5,5492 & 0,7759 \\
3- k=4 & 8,7911 & 2,1019 & 5,5435 & 0,7761 \\
4- k=5 & 8,7785 & 2,1046 & 5,5356 & 0,7764 \\
5- k=6 & 8,7645 & 2,1008 & 5,5267 & 0,7768 \\
6- k=7 & 8,7582 & 2,0990 & 5,5227 & 0,7770 \\
\hline
\end{tabular}



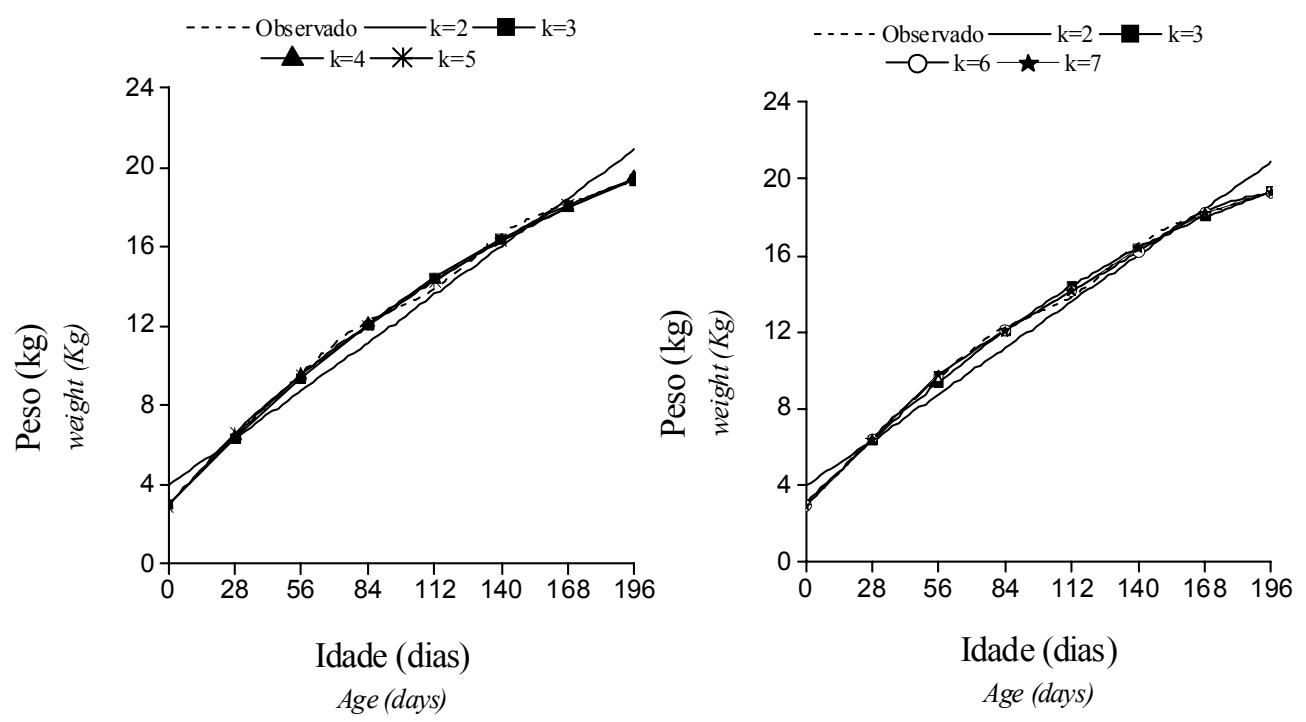

Figura 1. Curvas de crescimento médio, estimadas por funções polinomiais de segunda a sétima ordem. (Average growth curve, estimates for polynomial functions from second to seventh orders).

foram encontrados por Meyer (1999) e Sakaguti et al. (2002) em dados de bovinos.

Observando as curvas de crescimento dos animais, obtidas por funções polinomiais do peso dos animais em relação a suas idades (figura 1), nota-se que houve leve desaceleração do crescimento após os 112 dias de idade que pode ser atribuída ao estresse causado pelo desaleitamento, muitas vezes coincidindo com o período de baixa disponibilidade de alimento.

Os pesos dos animais em relação às suas idades, descritas por funções polinomiais de ordem três a sete, produziram curvas de crescimento semelhantes. Entre as funções analisadas, a linear foi a que apresentou trajetória de crescimento menos condizente com o desenvolvimento corporal dos animais, principalmente nas primeiras e últimas fases consideradas. Entre as funções que produziram curvas semelhantes, tornase evidente e mais adequada a utilização de uma função cúbica, uma vez que além de melhor ajuste, conforme critérios já apresentados, é menos parametrizada.
Todos os critérios utilizados (Log L, AIC e BIC) indicaram melhorias até a ordem

Tabela II. Ordem do polinômio para a regressão fixa $\left(K_{\beta}\right)$, efeitos genético direto $(K=3)$ e materno $\left(K_{m}=3\right)$, efeitos de ambiente permanente do animal $\left(K_{c}=3\right)$ e critérios logaritmo da função de máxima verossimilhança (Log L), informação de Akaike (AIC) e Bayesiano de Schwarz (BIC). (Order of polinomial for fixed regression $\left(\mathrm{K}_{\beta}\right)$, direct additive-genetic $\left(K_{a}=3\right)$ and maternal genetic effects $\left(K_{m}=3\right)$ and animal permanent environmental effects $\left(K_{c}=3\right)$ and maximum log likelihood criterion (LogL), Aikaike's information criterion (AIC) and Bayesian information criterion (BIC)).

\begin{tabular}{lccc}
\hline & \multicolumn{3}{c}{ Critérios } \\
$\mathrm{K}_{\beta}$ & Log L & AIC & BIC \\
\hline 2 & $-3741,25$ & 7520,51 & 7641,52 \\
3 & $-3691,24$ & 7420,49 & 7541,51 \\
4 & $-3687,37$ & 7412,74 & 7533,76 \\
5 & $-3688,48$ & 7414,96 & 7535,48 \\
6 & $-3687,38$ & 7412,76 & 7533,78 \\
7 & $-3687,38$ & 7412,76 & 7533,78 \\
\hline
\end{tabular}




\section{AVALIAÇÃO DA TRAJETÓRIAMÉDIA DE CRESCIMENTO DE CAPRINOS}
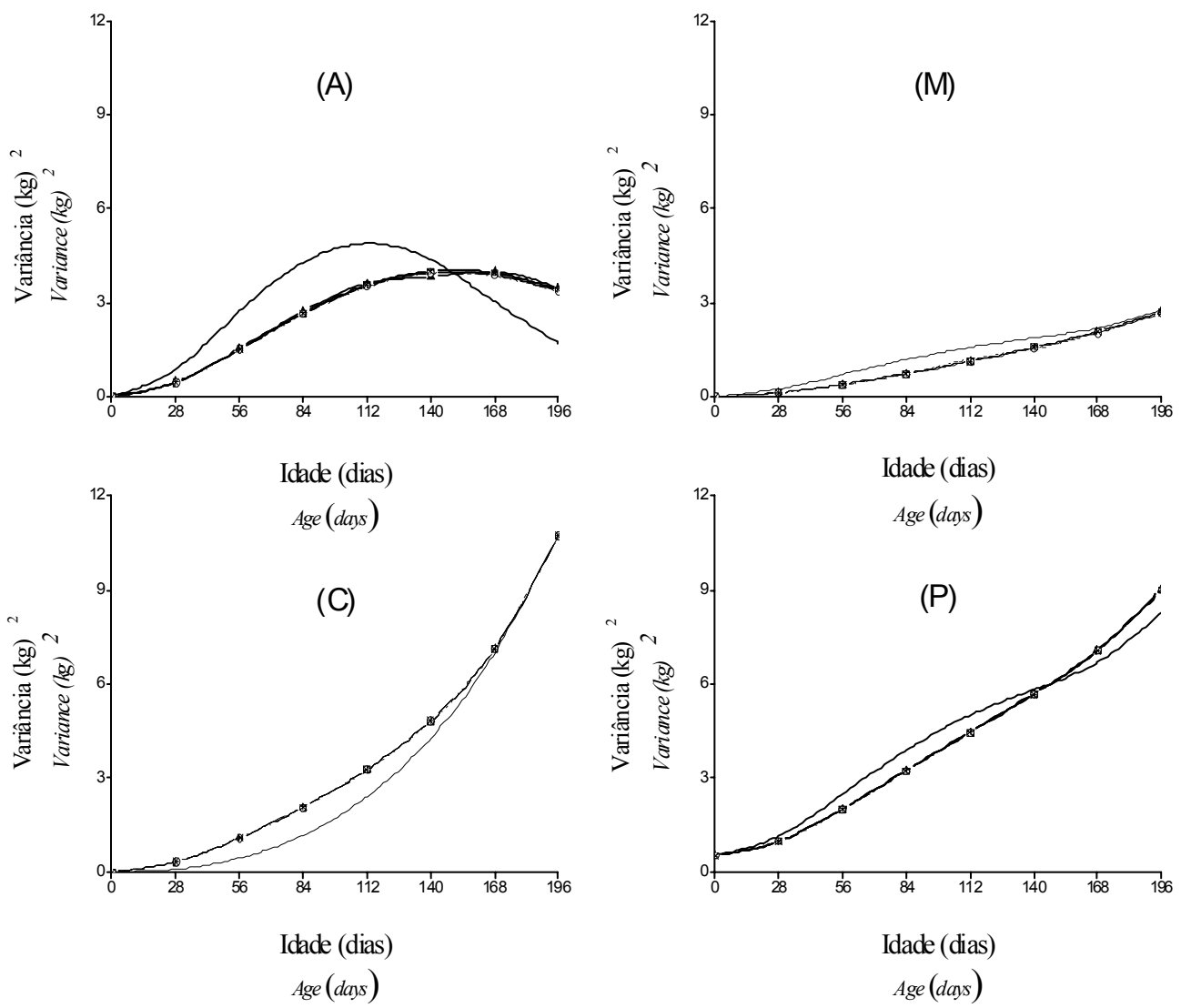

Idade (dias)

$\operatorname{Age}($ days $)$

$(\cos )$

Figura 2. Estimativas de componentes de variância genética aditiva direta (A), aditiva materna $(M)$, ambiente permanente de animal (C), e fenotípica, dividida por dois $(P)$ para as funções polinomiais de ordens: ( $-k=2$; $k=3$; $\Delta k=4$; o $k=5$; * $k=6$ e ---- $k=7$ ). (Estimates of direct additive-genetic (A), maternal genetic (M), animal permanent environmental $(C)$ and phenotypic, divided by two $(P)$ variance, for polynomial functions of orders: $(-k=2 ;-k=3 ; \boldsymbol{k}=4$; $o \mathrm{k}=5$; $*$ $\mathrm{k}=6 \mathrm{e}---\mathrm{k}=7)$ ).

de ajuste quatro, reforçando o melhor ajuste desta curva, obtido quando o efeito de animal foi desconsiderado, no estudo de crescimento de caprinos Anglo-Nubiano. Mais estudos são necessários para avaliar ajustes de funções da trajetória fixa de crescimento, uma vez que a ordenação quanto ao melhor ajuste pode variar entre estudos e populações.

A influência da ordem de ajuste da regressão fixa sobre os componentes de variância e os parâmetros genéticos, bem como os resultados relacionados aos critérios de adequação utilizados são apresentados na tabela II. Verifica-se que o ajuste de ordem quatro foi novamente o que melhor representou a trajetória fixa da população, agora considerando um polinômio de ordem três para os efeitos aleatórios genético aditivo direto, genético materno e ambiente permanente de animal.

As estimativas das variâncias genética aditiva direta e materna, de ambiente permanente de animal e fenotípica dividida por 
dois (para efeito de escala), estão apresentadas na figura 2. As variâncias genética aditiva direta (figura 2A) representadas pelas ordens de três a sete, apresentaram comportamento semelhantes em toda a trajetória de crescimento dos animais. Apresentaram valores muito baixo no início das pesagens, principalmente para peso ao nascimento, e foram aumentando até os 140 dias com leve redução aos 168 e 196 dias de idade. Estimativas de variância genética aditiva direta decrescentes do nascimento aos 150 dias de idade também foram observadas (Lewis e Brotherstone, 2002).

Os valores de baixa magnitude, próximos de zero ao nascimento, podem ser atribuídos à falta de ajustes da variância residual. Segundo Meyer (2001) a inclusão do peso ao nascer nas análises aumentou significativamente a ordem de ajuste requerida pelos modelos de regressão aleatória e os modelos de regressão aleatória podem apresentar dificuldades ao ajustarem dados que contenham grandes efeitos de escala. Os pesos ao nascer podem ser acompanhados por variações inexplicáveis, como fatores relacionados normalmente ao momento do nascimento. A função de ordem dois foi a que apresentou trajetória com maior diferença em relação às outras em todas as variâncias, o que pode ser indício de que esta função não representa bem o padrão de crescimento dos animais.

Estimativas de variâncias de ambiente permanente direto (figura 2C) e fenotípica dividida por dois (figura 2P) aumentaram à medida que o animal cresceu e praticamente não houve diferença nas trajetórias obtidas pelos diferentes modelos. Meyer (2001) descreveu padrão semelhante para pesos do nascimento à desmama de animais Hereford. Tanto o efeito permanente de animal como as variâncias fenotípicas, mostraram ser funções da idade do animal, dessa forma, as funções contínuas podem ser consideradas nos modelos animais em determinados períodos de crescimento.

As estimativas de herdabilidade estão apresentadas na figura 3. As herdabilidades direta e materna (figura 3A e $\mathbf{3} \mathbf{M}$ ) estimadas quando se ajustou função linear foram maiores que as estimadas pelas demais funções em toda a trajetória, exceto nas extremidades da curva, onde os valores das estimativas foram próximos.

Estimativas de herdabilidades diretas mais altas foram observadas entre 56 e 112 dias de idade, conforme o ajuste empregado. A estimativa de herdabilidade mais alta foi observada aos 84 dias de idade, sendo 0,55 para a função linear e 0,41 , para as demais ordens. Os valores obtidos em ambos os modelos para peso ao nascer e aos 196 dias foram de baixa magnitude, porém com rápido crescimento do nascimento aos 84 dias de idade, apresentando, a seguir, leve redução com o avanço da idade. E provável que esta redução seja atribuída ao pequeno número de informações nas idades, a partir dos 112 dias de idade e da eliminação de animais que apresentam baixo desempenho, em idades mais novas. Sakaguti et al. (2003) observaram resultado semelhante para herdabilidade em bovinos de corte.

A estimativa de herdabilidade materna foi baixa em todas as idades $(<0,18)$, apresentou leve aumento até os 56 dias de idade, mantendo-se constante à partir desta idade. Meyer (2001), ao usar modelos de regressão aleatória para duas raças de gado de corte na Austrália, também descreveu comportamento semelhante. Sarmento et al. (2006), em estudo com ovinos da raça Santa Inês, observaram tendência de declínio da herdabilidade materna nas últimas idades estudadas, mas aos 196 dias de idade houve, ainda, contribuição do efeito materno, em torno de $7 \%$ da variação fenotípica.

Estimativas das variâncias de ambiente permanente de animal como proporção das variâncias fenotípicas (figura 3C), apresentaram comportamentos diferentes de acordo com a ordem de ajuste. Pode-se observar que a função linear apresentou valores menores do que as demais funções nas idades intermediárias, enquanto nas

Archivos de zootecnia vol. 59, núm. 226, p. 274. 


\section{AVALIAÇÃO DA TRAJETÓRIAMÉDIA DE CRESCIMENTO DE CAPRINOS}
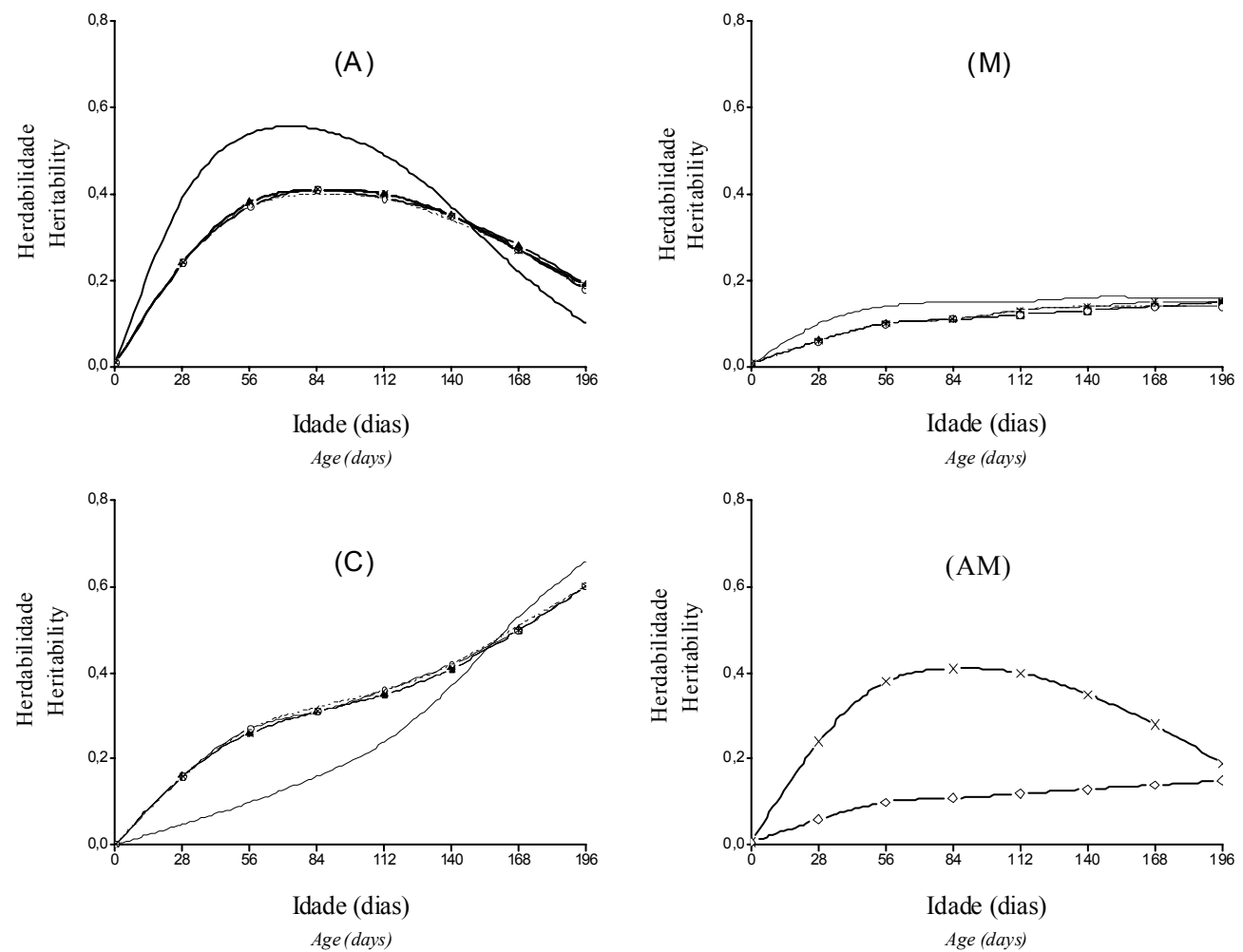

Figura 3. Estimativas de herdabilidade direta (A), materna (M), efeitos de ambiente permanente de animal como proporção da variância fenotípica $(C)$, para as funções polinomiais de ordens: ( $\quad k=2 ;-k=3 ; \boldsymbol{\Delta} k=4 ;$; $k=5$; * $k=6$; ---- $k=7)$ e (AM) herdabilidade direta $(x)$ versus materna $(\diamond)$ avaliada sobre uma função de ordem quatro. (Estimates of direct $(\mathrm{A})$ and maternal $(\mathrm{M})$ heritabilities, animal permanent environmental effects $(\mathrm{C})$, for Polynomial functions of orders: (- $-2=2 ;-\mathrm{k}=3 ; \boldsymbol{\Delta} \mathrm{k}=4 ; o \mathrm{k}=5 ; * \mathrm{k}=6$; ---- $\mathrm{k}=7$ ), and (AM) direct $(\mathrm{x})$ versus maternal $(\vartheta)$ herdabilidade avaliate by function of order four).

extremas foram próximas. Todas as funções, exceto a linear, apresentaram aumento maior do nascimento aos 56 dias de idade, e depois continuaram aumentando em forma suave.

Estimativas de herdabilidades direta versus materna, obtidas por meio de função cúbica, são apresentadas na figura $\mathbf{3 A M}$. Os efeitos maternos apresentaram menor influência que os aditivos diretos em todas as idades, em decorrência do aleitamento adotado para os animais na estação experimental, com vistas à prevenção da CAE artrite-encefalite caprina.
Os resultados divergem, em parte, dos obtido por Sarmento et al., 2006, que verificaram maior herdabilidade materna nas pesagens iniciais, com as herdabilidades aditivas diretas apresentando comportamento crescente do nascimento ao $196^{\circ}$ dia de idade. É provável que a trajetória decrescente da herdabilidade direta obtida, seja atribuída ao pequeno número de observações nas últimas idades, uma vez que os polinômios ortogonais de Legendre apresentam dificuldade em ajustar adequadamente dados desta natureza (Kirkpatrick et al., 1994) 


\section{SOUSA, SILVA, SARMENTO, SOUSAE SOUZA}

Os resultados obtidos poderão fornecer subsídios para os programas de melhoramento de caprinos destinados a produção de carne, haja vista que a determinação da função que melhor representa a trajetória média de crescimento da espécie, é de fundamental importância na modelagem dos efeitos aleatórios de forma mais adequada, possibilitando assim obtenção de avaliações genéticas mais acuradas.

Em concluçao as funções polinomiais é

\section{BIBLIOGRAFIA}

Ali, T.E. and Schaeffer, R. 1987. Accounting for covariances among test day milk yields in dairy cows. Can. J. Anim. Sci., 67: 637- 644.

Arango, J.A., Cundiff, L.V. and Van Vleck, L.D. 2004. Covariance functions and random regression models for cow weight in beef cattle. J. Anim. Sci., 82: 54-67.

Assis, G.M.L., Albuquerque, L.G., Sarmento, J.L.R., Carneiro Júnior, J.M., Lopes, P.S. e Rodrigues, M.T. 2006. Estimação de parâmetros genéticos em caprinos leiteiros por meio de análise de regressão aleatória utilizando-se a amostragem de Gibbs. Rev. Bras. Zootecn., 35: 706-714.

Breda, F.C., Albuquerque, L.G., Yamaki, M., Reis Filho, J.C., Sarmento, J.L.R, Lopes, P.S. e Rodrigues, M.T.2006. Estimação de parâmetros genéticos para produção de leite de cabras da raça Alpina. Rev. Bras. Zootecn., 35: 396-404.

Kirkpatrick, M., Lofsvold, D. and Bulmer, M. 1990. Analysis of the inheritance, selection and evolution of growth trajectories. Genetics, 124: 979-993.

Kirkpatrick, M., Hill, W.G. and Thompson, R. 1994. Estimating the covariance structure of traits during growth and aging, illustrated with lactations in dairy cattle. Genet. Res., 64: 57-69.

Lewis, R.M. and Brotherstone, S. 2000. A genetic evaluation of growth in sheep using random regression techniques. J. Anim. Sci., 74: 6370.

Meyer, K. 1998. "DXMRR" - a program to estimate covariance functions for longitudinal data by restricted maximum likelihood. In: World Congress eficiente e polinômios de ordem quatro devem ser empregadas para representar a curva média de crescimento de caprinos Anglonubiano, em modelos de regressão aleatória.

$\mathrm{O}$ ajuste da regressão fixa apresentou influência sobre as estimativas dos parâmetros genéticos, indicando a necessidade de se modelar adequadamente a curva média de crescimento de caprinos da raça AngloNubiana, por meio de modelos de regressão aleatória.

on Genetics Applied to Livestock Production, 6. Proceedings... Armidale. V. 25, pp. 517-520.

Meyer, K. 1999. Estimates of genetic and phenotypic covariance functions for postweaning growth and mature weight of beef cows. J. Anim. Breed. Genet., 116: 181-205.

Meyer, K. 2001. Estimates of direct and maternal covariance functions for growth of Australian beef calves from birth to weaning. Genet. Sel. Evol., 33: 1-28.

Sakaguti, E.S., Silva, M.A. e Martins, E.N. 2002. Trajetória de crescimento e efeito da idade da vaca nos modelos de regressão aleatória de bovinos da raça Tabapuã. Arq. Bras. Med. Vet. Zootecn., 54: 414-423.

Sakaguti, E.S., Silva, M.A. e Quaas, R.L. 2003. Avaliação do crescimento de bovinos jovens da raça Tabapuã, por meio de análise de funções de covariância. Rev. Bras. Zootecn. 32: 864874.

Sarmento, J.L.R., Torres, R.A., Pereira, C.S., Sousa, W.H., Lopes, P.S, Araújo, C.V. e Euclydes, R.F. 2006. Avaliação genética de características de crescimento de ovinos Santa Inês utilizando modelos de regressão aleatória. Arq. Bras. Med. Vet. Zootecn., 58: 68-77.

SAS Institute. 1999. USER'S guide: statistics. Version 8.0. SAS Institute. Cary, NC.

Sousa, J.E.R. 2007. Avaliação do crescimento de caprinos da raça Anglo-nubiana usando-se regressão aleatória. Tese (Doutorado em Ciência Animal). Universidade Federal de Minas Gerais. Belo Horizonte. 72 pp.

Archivos de zootecnia vol. 59, núm. 226, p. 276. 